\title{
Blood-based epigenetic estimators of chronological age in human adults using DNA methylation data from the lllumina MethylationEPIC array
}

Yunsung Lee (1,2* $^{*}$, Kristine L. Haftorn ${ }^{1,2,3}$, William R. P. Denault ${ }^{1,3,4}$, Haakon E. Nustad ${ }^{3,5}$, Christian M. Page ${ }^{3,6}$, Robert Lyle ${ }^{3,7,8}$, Sindre Lee-Ødegård ${ }^{9,10}$, Gunn-Helen Moen ${ }^{11,12,13,14}$, Rashmi B. Prasad ${ }^{15}$, Leif C. Groop ${ }^{15,16}$, Line Sletner ${ }^{17,18}$, Christine Sommer ${ }^{19}$, Maria C. Magnus 3,14,20, Håkon K. Gjessing ${ }^{3,4}$, Jennifer R. Harris ${ }^{1,3}$, Per Magnus ${ }^{3}$, Siri E. Håberg ${ }^{3}$, Astanand Jugessur ${ }^{1,3,4+}$ and Jon Bohlin $3,21 \dagger$

\begin{abstract}
Background: Epigenetic clocks have been recognized for their precise prediction of chronological age, age-related diseases, and all-cause mortality. Existing epigenetic clocks are based on CpGs from the Illumina HumanMethylation450 BeadChip (450 K) which has now been replaced by the latest platform, Illumina MethylationEPIC BeadChip (EPIC). Thus, it remains unclear to what extent EPIC contributes to increased precision and accuracy in the prediction of chronological age.

Results: We developed three blood-based epigenetic clocks for human adults using EPIC-based DNA methylation (DNAm) data from the Norwegian Mother, Father and Child Cohort Study (MoBa) and the Gene Expression Omnibus (GEO) public repository: 1) an Adult Blood-based EPIC Clock (ABEC) trained on DNAm data from MoBa ( $n=1592$, agespan: 19 to 59 years), 2) an extended ABEC (eABEC) trained on DNAm data from MoBa and GEO ( $n=2227$, age-span: 18 to 88 years), and 3) a common $A B E C$ ( $C A B E C)$ trained on the same training set as eABEC but restricted to CpGs common to $450 \mathrm{~K}$ and EPIC. Our clocks showed high precision (Pearson correlation between chronological and epigenetic age $(r)>0.94)$ in independent cohorts, including GSE111165 ( $n=15)$, GSE115278 $(n=108)$, GSE132203 $(n=$ 795), and the Epigenetics in Pregnancy (EPIPREG) study of the STORK Groruddalen Cohort $(n=470)$. This high precision is unlikely due to the use of EPIC, but rather due to the large sample size of the training set.
\end{abstract}

Conclusions: Our ABECs predicted adults' chronological age precisely in independent cohorts. As EPIC is now the dominant platform for measuring DNAm, these clocks will be useful in further predictions of chronological age, agerelated diseases, and mortality.

Keywords: DNA methylation, Epigenetic age, Chronological age, Illumina MethylationEPIC BeadChip, MoBa

\footnotetext{
* Correspondence: Yunsung.Lee@fhi.no

Astanand Jugessur and Jon Bohlin are Joint last authors

'Department of Genetics and Bioinformatics, Norwegian Institute of Public Health, Oslo, Norway

${ }^{2}$ Institute of Health and Society, Faculty of Medicine, University of Oslo, Oslo, Norway

Full list of author information is available at the end of the article
}

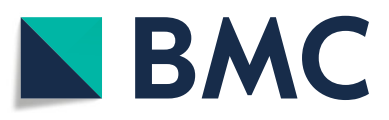

( The Author(s). 2020 Open Access This article is licensed under a Creative Commons Attribution 4.0 International License, which permits use, sharing, adaptation, distribution and reproduction in any medium or format, as long as you give appropriate credit to the original author(s) and the source, provide a link to the Creative Commons licence, and indicate if changes were made. The images or other third party material in this article are included in the article's Creative Commons licence, unless indicated otherwise in a credit line to the material. If material is not included in the article's Creative Commons licence and your intended use is not permitted by statutory regulation or exceeds the permitted use, you will need to obtain permission directly from the copyright holder. To view a copy of this licence, visit http://creativecommons.org/licenses/by/4.0/. The Creative Commons Public Domain Dedication waiver (http://creativecommons.org/publicdomain/zero/1.0/) applies to the data made available in this article, unless otherwise stated in a credit line to the data. 


\section{Background}

Aging is a biological phenomenon that is characterized by reduced functional capacity $[1,2]$. Because chronological age is an imperfect surrogate of aging [3-6], the concept of biological aging that can capture the different rate of functional deterioration across individuals has been suggested [1]. Given the significance of biological aging, a variety of predictors of biological age have been constructed based on known hallmarks of aging $[6,7]$, including telomere length [8], metabolic rate [9], DNA methylation (DNAm) [10], $\mathrm{CD} 4+$ and $\mathrm{CD} 8+\mathrm{T}$ cell ratio [11], proteomic alterations [12], and gut microbiota [13]. Among these, DNAm-based estimators of chronological age (referred to as epigenetic clocks) have garnered the most interest due to their remarkable precision in estimating chronological age, agerelated diseases, and all-cause mortality [4, 14-18].

Epigenetic age is a linear combination of DNAm levels at specific CpGs, which are weighted by their respective coefficients estimated through an epigenetic clock. Most of the previously published epigenetic clocks (the Hannum Blood-based clock [19], Horvath Pan-tissue clock [20], Levine PhenoAge clock [16], and Horvath Skin \& Blood clock [3]) were based on specific CpGs from the Illumina HumanMethylation450 BeadChip (450 K). This platform has recently been replaced by the Illumina MethylationEPIC BeadChip (EPIC). EPIC is a major improvement over its predecessor, $450 \mathrm{~K}$ (> 450,000 CpGs), in terms of the number of probes (>850,000 CpGs) and the genomic coverage of regulatory elements [21]. To our knowledge, only one EPIC-based epigenetic clock has been published (the Alsaleh EPIC clock [22]). This clock was trained on a relatively small training set and was not sufficiently validated in independent cohorts. Thus, it remains unclear to what extent EPIC contributes to increased precision and accuracy in the prediction of chronological age.

We developed three blood-based epigenetic clocks for human adults: 1) an Adult Blood-based EPIC Clock (ABEC) trained on EPIC-derived DNAm data from adult peripheral blood in a sub-study of the Norwegian Mother, Father and Child Cohort Study (MoBa) [23] called the STudy of Assisted Reproductive Technology (MoBa-START); 2) an extended ABEC (eABEC) trained on MoBa-START and publicly available DNAm data from the Gene Expression Omnibus (GEO) with the aim of improving the performance of $\mathrm{ABEC}$; and 3) a common ABEC (cABEC) trained on the same training set as eABEC but restricted to CpGs common to $450 \mathrm{~K}$ and EPIC. The purpose of cABEC was to determine whether the additional CpGs on EPIC improved predictions of chronological age. We validated our clocks and the other published clocks (the Hannum Blood-based clock, Horvath Pan-tissue clock, Levine PhenoAge clock, Horvath Skin \& Blood clock, Alsaleh EPIC clock, and Zhang clock) in EPIC-derived DNAm data from independent cohorts, including publicly available DNAm data from GEO and the Epigenetics in Pregnancy (EPIPREG) study of the STORK Groruddalen Cohort (STORK) [24].

\section{Results}

\section{Peripheral blood-based DNA methylation}

We trained an epigenetic clock using elastic net regression on DNAm data from 1592 adults who were mothers and fathers in MoBa-START (796 women and 796 men). The chronological age of these adults ranged from 19 to 59 years (19 to 46 years for women and 19 to 59 years for men). DNAm on these individuals was measured using EPIC. For the current analyses, we focused on the 770,586 autosomal CpGs that remained after quality control (see Methods). Table 1 provides additional details regarding the MoBa-START samples.

\section{Adult blood-based EPIC clock (ABEC)}

Figure 1 summarizes our analysis flow.

We developed ABEC using a blood-based DNAm dataset consisting of adults (training set $n=1592$, Table 1, Fig. 1). We used elastic net regression [32] to select the most predictive $\mathrm{CpGs}$ for chronological age. The resulting regression comprised 1695 CpGs. The predicted DNAm age was calculated using the following equation:

$$
\begin{aligned}
D N A m \text { Age }_{j}= & \hat{\beta}_{(\text {Intercept })}+X_{c g 1, j} \hat{\beta}_{c g 1}+X_{c g 2, j} \hat{\beta}_{c g 2}+\ldots \\
& +X_{c g 1695, j} \hat{\beta}_{c g 1695},
\end{aligned}
$$

where DNAm Age $e_{j}$ is the epigenetic age of the $j$ th individual, and $X_{c g i, j}$ refers to the DNAm level of the $j$ th individual at the $i$ th CpG site. The estimated intercept and beta coefficients are provided in Supplementary File 1.

Figure 2 shows the performance of ABEC in the training set $(n=1592$, Fig. $2 \mathrm{a})$ and the test set $(n=424$, Fig. $2 \mathrm{~b})$. The prediction precision was quantified using the Pearson correlation coefficient $(r)$ between DNAm age and chronological age. The prediction accuracy was quantified using the median absolute deviation (MAD) between DNAm age and chronological age. ABEC showed high precision and accuracy in both of the training $(r=0.999, M A D=0.14$, Fig. 2a) and test set $(r=0.95, M A D=1.13$, Fig. $2 b)$. The red line in Fig. $2 \mathrm{a}$ and $\mathrm{b}$ represents a perfect correlation between chronological age and DNAm age, and the dotted line refers to the regression of the predicted DNAm age on chronological age.

Despite its overall high precision, ABEC slightly underestimated the age of the older individuals, particularly those above 45 years of age (Fig. 2c, d). This bias is expected given that the MoBa-START dataset is a pregnancy cohort with few individuals older than 45 years. In addition, most individuals aged 45 years or older were 
Table 1 Description of the peripheral whole-blood-derived DNAm data on the EPIC platform

\begin{tabular}{|c|c|c|c|c|c|c|c|}
\hline Cohort & Tissue type & Platform & GEO submitter & $N$ & $\begin{array}{l}\text { Normalization } \\
\text { Method }^{\mathrm{a}}\end{array}$ & $\begin{array}{l}\text { Probe exclusion } \\
\text { Criteriab }^{b}\end{array}$ & $\begin{array}{l}\text { Age range } \\
\text { (years) }\end{array}$ \\
\hline \multicolumn{8}{|l|}{ ABEC } \\
\hline \multicolumn{8}{|l|}{ Training data } \\
\hline $\begin{array}{l}\text { MoBa-STAR } \\
T\end{array}$ & $\begin{array}{l}\text { Peripheral whole } \\
\text { blood }\end{array}$ & EPIC & - & 1592 & BMIQ & $\mathrm{SC}, \mathrm{CH}, \mathrm{DP}, \mathrm{SNP}$ & $19-59$ \\
\hline \multicolumn{8}{|l|}{ Test data } \\
\hline $\begin{array}{l}\text { MoBa-STAR } \\
T\end{array}$ & $\begin{array}{l}\text { Peripheral whole } \\
\text { blood }\end{array}$ & EPIC & - & 424 & BMIQ & $\mathrm{SC}, \mathrm{CH}, \mathrm{DP}, \mathrm{SNP}$ & $20-58$ \\
\hline \multicolumn{8}{|l|}{ eABEC } \\
\hline \multicolumn{8}{|l|}{ Training data } \\
\hline $\begin{array}{l}\text { MoBa-STAR } \\
T\end{array}$ & $\begin{array}{l}\text { Peripheral whole } \\
\text { blood }\end{array}$ & EPIC & - & 1592 & BMIQ & $\mathrm{SC}, \mathrm{CH}, \mathrm{DP}, \mathrm{SNP}$ & $19-59$ \\
\hline GSE116339 & $\begin{array}{l}\text { Peripheral whole } \\
\text { blood }\end{array}$ & EPIC & Curtis et al. [25] & 635 & Noob & SC & $23-88$ \\
\hline \multicolumn{8}{|l|}{ Test data } \\
\hline $\begin{array}{l}\text { MoBa-STAR } \\
T\end{array}$ & $\begin{array}{l}\text { Peripheral whole } \\
\text { blood }\end{array}$ & EPIC & - & 424 & BMIQ & $\mathrm{SC}, \mathrm{CH}, \mathrm{DP}, \mathrm{SNP}$ & $20-58$ \\
\hline GSE111165 & $\begin{array}{l}\text { Peripheral whole } \\
\text { blood }\end{array}$ & EPIC & $\begin{array}{l}\text { Shinozaki et al. } \\
\text { [26] }\end{array}$ & 15 & Noob & SC & $24-61$ \\
\hline GSE115278 & $\begin{array}{l}\text { Peripheral whole } \\
\text { blood }\end{array}$ & EPIC & Arpon et al. [27] & 108 & Noob & SC & $19-66$ \\
\hline \multicolumn{8}{|l|}{ Other test data } \\
\hline EPIPREG & $\begin{array}{l}\text { Peripheral whole } \\
\text { blood }\end{array}$ & EPIC & - & 470 & FunNorm & $\mathrm{SC}, \mathrm{CH}, \mathrm{DP}, \mathrm{SNP}$ & $19-42$ \\
\hline GSE132203 & $\begin{array}{l}\text { Peripheral whole } \\
\text { blood }\end{array}$ & EPIC & Kilaru et al. [28] & 795 & Noob & SC & $18-76$ \\
\hline
\end{tabular}

a Pre-processing method for quantifying DNAm levels in the range of 0 to 1

Noob Normal-exponential out-of-band [29]

$B M I Q$ Beta-mixture quantile dilation [30]

FunNorm Functional normalization [31]

b Probe exclusion criteria

SC Sex chromosome, $C H$ cross-hybridizing, DP detection $P$-value $<0.01$ and SNP single-nucleotide polymorphism

males, which may introduce a sex-bias in the prediction of chronological age.

\section{Extended adult blood EPIC clock (eABEC)}

To reduce the underestimation bias and improve the precision of ABEC among older individuals in the MoBa-START dataset, we developed an extended ABEC (eABEC) by adding a publicly available DNAm dataset, GSE116339 $(n=635)$ [25], from the GEO data repository (https://www.ncbi.nlm.nih.gov/geo/) [33] to the original training set for ABEC (Fig. 3). This increased the total sample size of the new training set to 2227. Elastic net regression was used in the same manner as for ABEC above, and for this training set, the number of selected CpGs was 1791.

We validated eABEC in an extended test set consisting of the test set for $\mathrm{ABEC}$ and two independent cohorts (GSE111165 and GSE115278) from GEO. We selected these GEO datasets because they were EPIC-derived blood-based DNAm data with a wide age span (20 to 70 years). The inclusion of GSE116339 substantially improved the prediction in individuals aged 45 years and above (Fig. 3a, b), but there was a slight underestimation of age among individuals aged 65 years or older in both the training and test set (Fig. 3c, d).

\section{Advantage of EPIC in developing epigenetic clocks}

One major difference between our epigenetic clocks (ABEC and eABEC) and the previously published clocks was the use of EPIC for the training set. The training set of the other epigenetic clocks was mostly based on 450 $\mathrm{K}$, except for the Horvath Skin \& Blood clock which used both $450 \mathrm{~K}$ and EPIC-derived DNAm data. To assess whether EPIC-derived DNAm data yield a more accurate and precise clock, we trained a third epigenetic clock using the same training set as for eABEC but using only the 397,473 autosomal CpG sites that are in common between EPIC and $450 \mathrm{~K}$. We refer to this third clock as 'common' ABEC (cABEC) hereafter. Elastic net regression selected $1892 \mathrm{CpG}$ sites. 


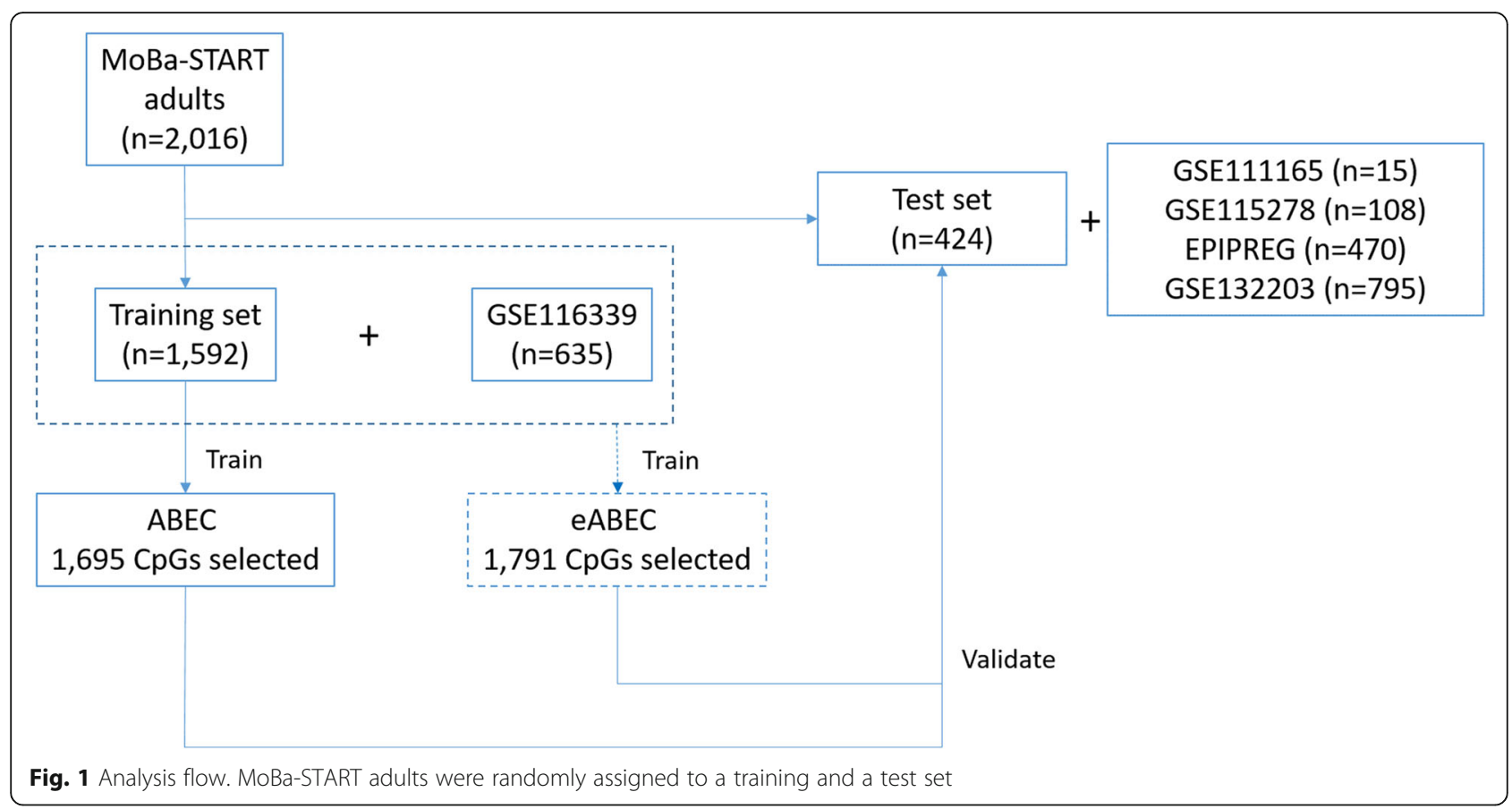

cABEC showed a high prediction performance, similar to eABEC (Supplementary File 2, S-Figure 1). The precision metric ( $r$ ) of cABEC was identical to that of eABEC. However, compared to eABEC, the accuracy of cABEC in the test set was slightly diminished (MAD $=1.25 \rightarrow 1.3$ ).

We hypothesized that the denser EPIC array might be beneficial in developing an epigenetic clock with a smaller training set. To address this point, two types of epigenetic clocks (one using all the CpGs on EPIC and the other using the CpGs common to EPIC and $450 \mathrm{~K}$ ) were trained on random subsets of the training set of eABEC and validated in the test sample of eABEC (see Methods for further details). Both types of epigenetic clocks showed a remarkable improvement in precision and accuracy as the sample size of the training set increased (Fig. 4). However, across all the reduced training sets, the epigenetic clock based on all the CpGs on EPIC did not outperform the other clock based on the CpGs common to EPIC and $450 \mathrm{~K}$ (Fig. 4). This indicates that the additional CpGs on EPIC do not enhance the accuracy or precision of the epigenetic clocks when the training set is reduced.

\section{Validation of ABECs and other epigenetic clocks}

Using an independent cohort from GEO $(n=123)$, we evaluated the performance of $A B E C$, eABEC, and cABEC against six published epigenetic clocks: the Hannum Blood-based clock [19], Horvath Pan-tissue clock [20], Levine PhenoAge clock [16], Horvath Skin \& Blood clock [3], Alsaleh EPIC clock [22], and Zhang clock [34]. The independent test set consisted of GSE111165 [26] and
GSE115278 [27] from the GEO database (see Table 1 for details). None of these GEO datasets have previously been used to train any epigenetic clocks.

Figure 5 summarizes the results of epigenetic age prediction by ABEC, eABEC, cABEC, and the six published epigenetic clocks mentioned above. Our eABEC and the Zhang clock showed the highest precision $(r=0.96)$, followed by ABEC $(r=0.95)$, cABEC $(r=0.95)$, the Horvath Skin \& Blood clock $(r=0.94)$, and the Hannum Blood-based epigenetic clock $(r=0.87)$. The 95\% confidence intervals of the $\mathrm{r}$ values can be found in Supplementary File 2 (S-Table 1 ). Here, we note that only the precision metric (r) was presented in Fig. 5 because the dots in the scatter plots could deviate systematically from the 45-degree line (so-called systematic offset) but still form a very tight prediction, e.g., panel (D) in Fig. 5. In such cases where high precision and relatively low accuracy are present, the systematic offset can be calibrated using a linear transformation, or, if necessary, a non-linear transformation.

An important distinction of ABECs from the other published clocks is that they are based on an ethnically homogeneous training set (MoBa-START and GSE116339 comprised individuals of European ancestry). We validated $A B E C$, eABEC, $c A B E C$, and the other published epigenetic clocks in the EPIC-derived blood-based DNAm data from EPIPREG $(n=470 ; 305$ European women and 165 South Asian women, Fig. 6), a sub-study of the STORK Groruddalen Cohort [24]. ABEC, eABEC, cABEC, the Horvath Skin \& Blood clock, and Zhang clock showed the highest precisions $(r>0.9)$. More interestingly, eABEC 


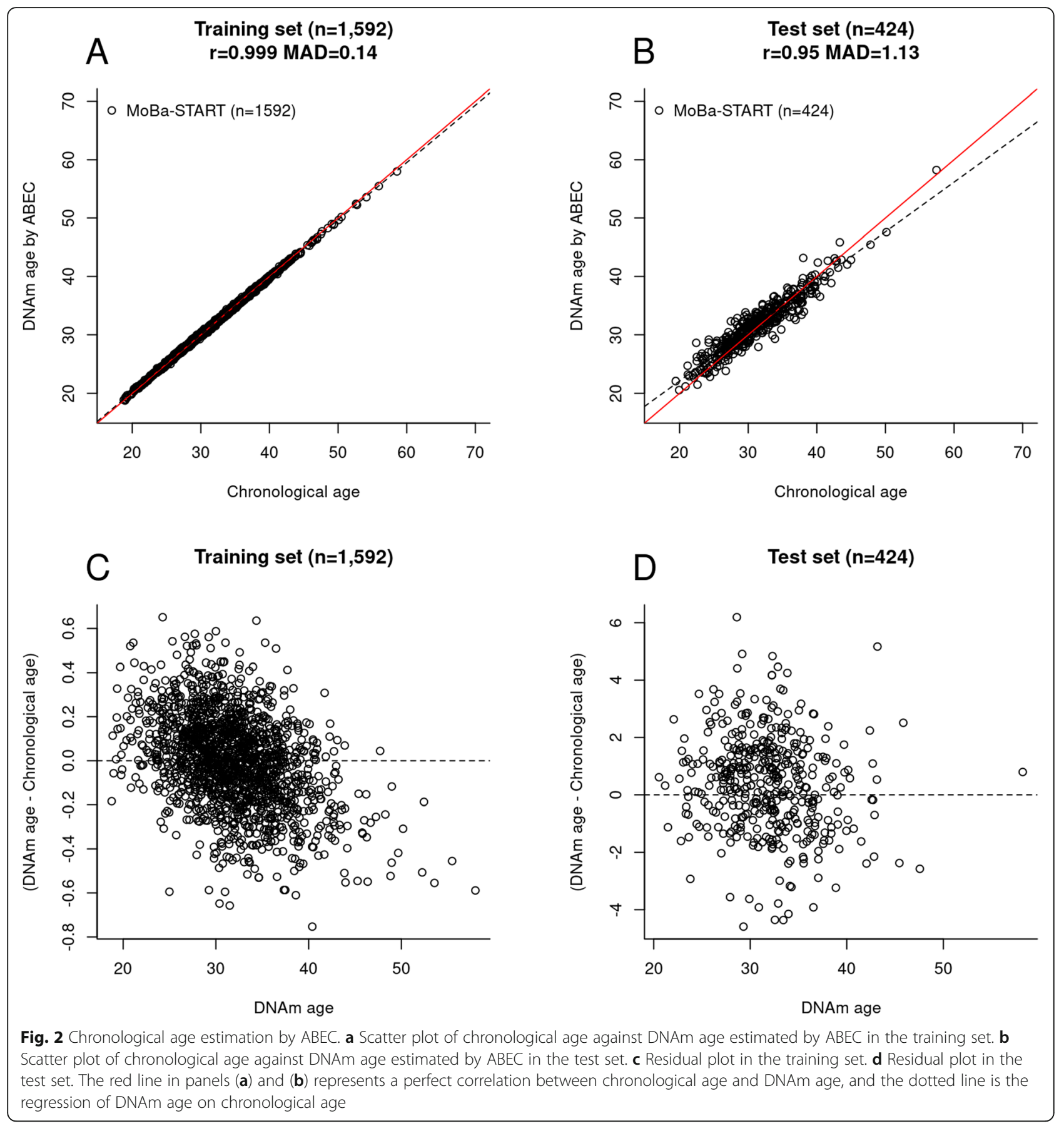

showed that the epigenetic age acceleration (EAA; residuals from the regression of DNAm age on chronological age) was higher in South Asian women than in Norwegian women $(+0.51$ years, $P=0.0015$, Supplementary File 2 , SFigure 2A). EAA derived by the Alsaleh EPIC clock was also elevated in South Asians compared to Norwegians (+ 0.25 years, $P=4 \mathrm{E}-04$, Supplementary File 2 , S-Figure $2 \mathrm{~B}$ ). However, EAAs derived by ABEC, cABEC, and the other published clocks did not show any difference between the two groups.
Given that $A B E C$, eABEC, and $\mathrm{CABEC}$ were trained on the ethnically homogeneous training set of Europeans, they may be sub-optimal for predicting chronological age in other ethnicities. To explore this further, we applied ABEC, eABEC, CABEC, and the other published epigenetic clocks to a GEO dataset comprising African Americans (GSE132203 [28]; $n=795$, Supplementary File 2, S-Figure 3). All the clocks, except for the Alsaleh EPIC clock, showed high correlations between chronological age and epigenetic age $(r>0.86)$. The 95\% 


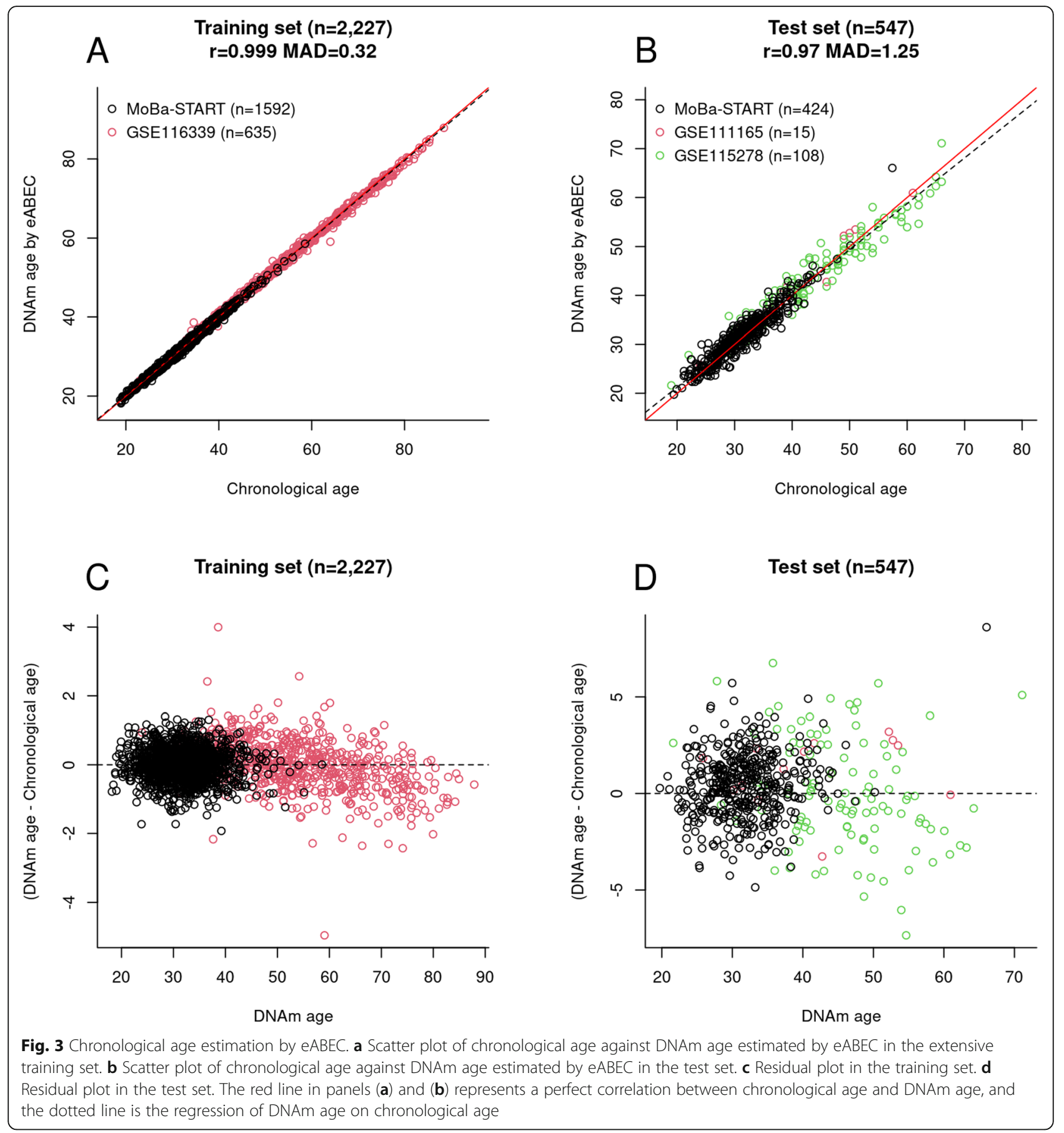

confidence intervals of the $r$ values can be found in Supplementary File 2 (S-Table 1). eABEC, cABEC, and the Zhang clock showed the highest $\mathrm{r}$ of 0.96 , and ABEC and the Horvath Skin \& Blood clock showed the secondhighest $r$ of 0.95 .

\section{Discussion}

We developed precise epigenetic clocks (ABEC and eABEC) using blood-based DNAm data from EPIC. Our epigenetic clocks showed a more precise chronological age prediction than existing blood-based epigenetic clocks (e.g., the Hannum Blood-based clock and Horvath Skin \& Blood clock; Fig. 5). The reason for the higher precision is more likely due to the large training set $(n=$ 2227, Table 1) and the wide age-span of the samples (19 to 88 years for the training set of eABEC, Table 1), which is consistent with the findings by Zhang and colleagues [34]. Compared to eABEC, both Hannum Blood- 


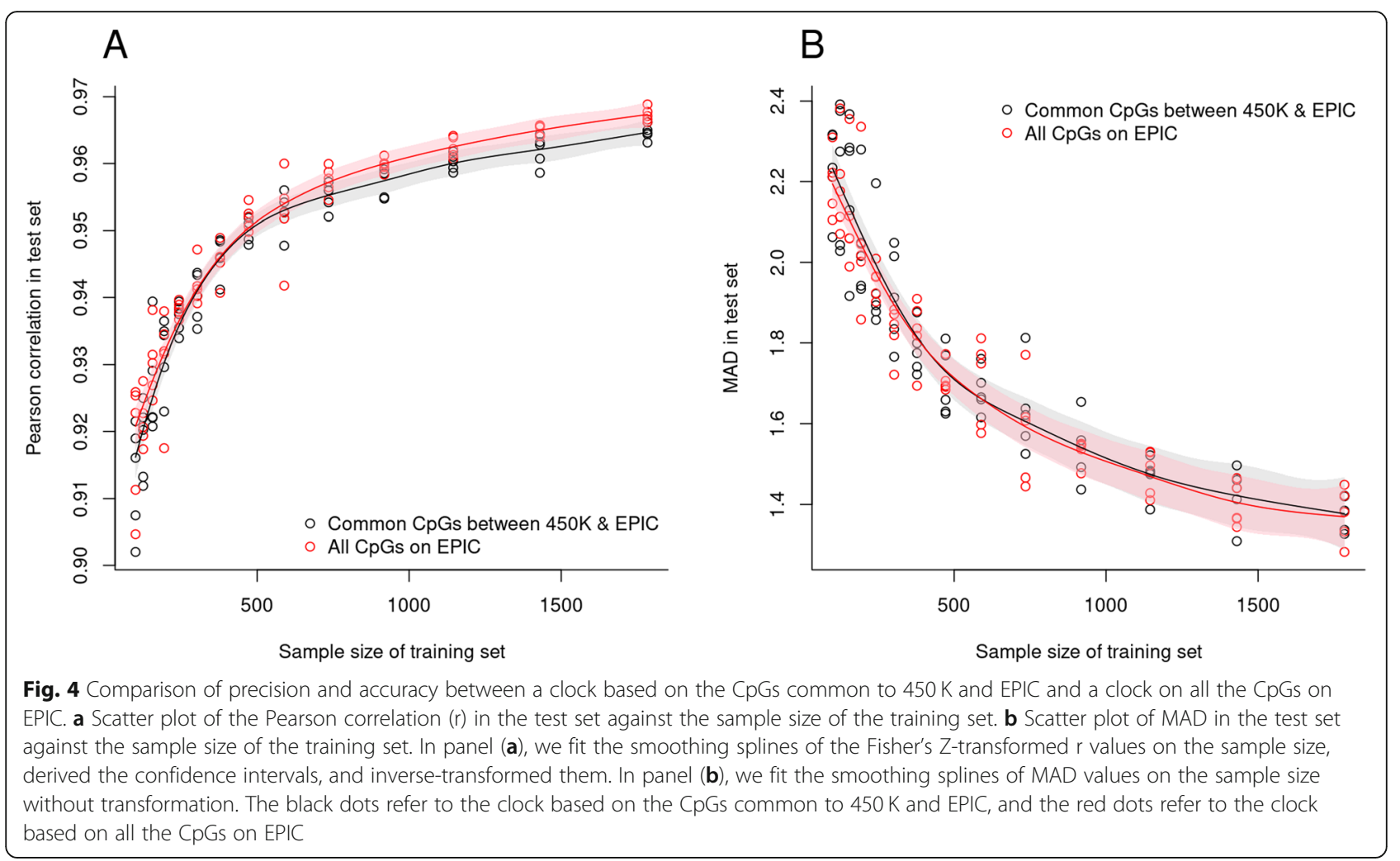

based clock and Horvath Skin \& Blood clock were trained on fewer samples $(n=656$ and $n=896$, respectively) that had a wider age-span (19 to 101 years and 0 to 94 years, respectively) [3, 19]. Other clocks (the Horvath Pan-tissue clock and Levine PhenoAge clock) may not be directly comparable to eABEC for chronological age prediction. For instance, the Horvath Pan-tissue clock was designed to measure epigenetic aging not only in blood but in multiple tissues [20], and the Levine PhenoAge was designed to predict phenotypic age (estimated using 10 clinical biomarkers, e.g., albumin, creatinine, serum glucose, and seven others) based on DNAm [16].

To develop eABEC, we added GSE116339 to the training set of ABEC. GSE116339 is from a study by Curtis et al. [25] that used EPIC to measure DNAm in peripheral blood samples collected from 658 individuals of European ancestry (638 non-Hispanic and 20 Hispanic) in Michigan, USA. These individuals had been exposed to the endocrine-disrupting chemical polybrominated biphenyl when an agricultural accident introduced it into the food supply in the 1970s. We selected 635 individuals from the control group whose total PBB (PBB-153, PBB-101, PBB-77, and PBB180) exposure was lower than $5 \mathrm{pg} / \mathrm{ml}$. The distribution of the total PBB exposure was highly right-skewed.

The high precision of eABEC cannot be attributed solely to the use of the EPIC platform as the additional 413,743 CpGs on EPIC did not improve age prediction noticeably (Fig. 4). Although the $1791 \mathrm{CpGs}$ selected by eABEC included 1084 CpGs that only exist on EPIC, eABEC did not outperform cABEC that used the CpGs common to $450 \mathrm{~K}$ and EPIC. This indicates that 226,915 probes (out of 413,743 ) that are designed to cover regulatory regions (DNase proximal/distal [35] and FANTOM5 [36]) did not increase the precision of the epigenetic clocks significantly [21]. Yet, Pidsley et al. [21] reported that probes on EPIC cover 58\% of FANTOM5 enhancers, $7 \%$ of distal, and $27 \%$ of proximal ENCODE regulatory regions, suggesting that the coverage of regulatory regions is still low. Thus, it is difficult to dismiss the possibility that other regulatory CpGs not currently included on EPIC might improve age prediction.

Underestimation and overestimation of epigenetic clocks should be carefully assessed using residual plots instead of scatter plots. As we regressed chronological age on DNAm levels (chronological age $=$ DNAm levels + error), a scatter plot that displays chronological age on the $\mathrm{x}$-axis and DNAm age on the $\mathrm{y}$-axis may lead to the misconception that DNAm age is overestimated in the oldest age group and underestimated in the youngest age group (Supplementary File, S-Figure 4). In contrast, residual plots that display DNAm age on the $\mathrm{x}$-axis and residuals (DNAm age minus chronological age) on the $y$ axis would enable a fair evaluation of prediction models. The strength of the current scatter plots lies in the visualization of EAA (the residuals of the regression of 


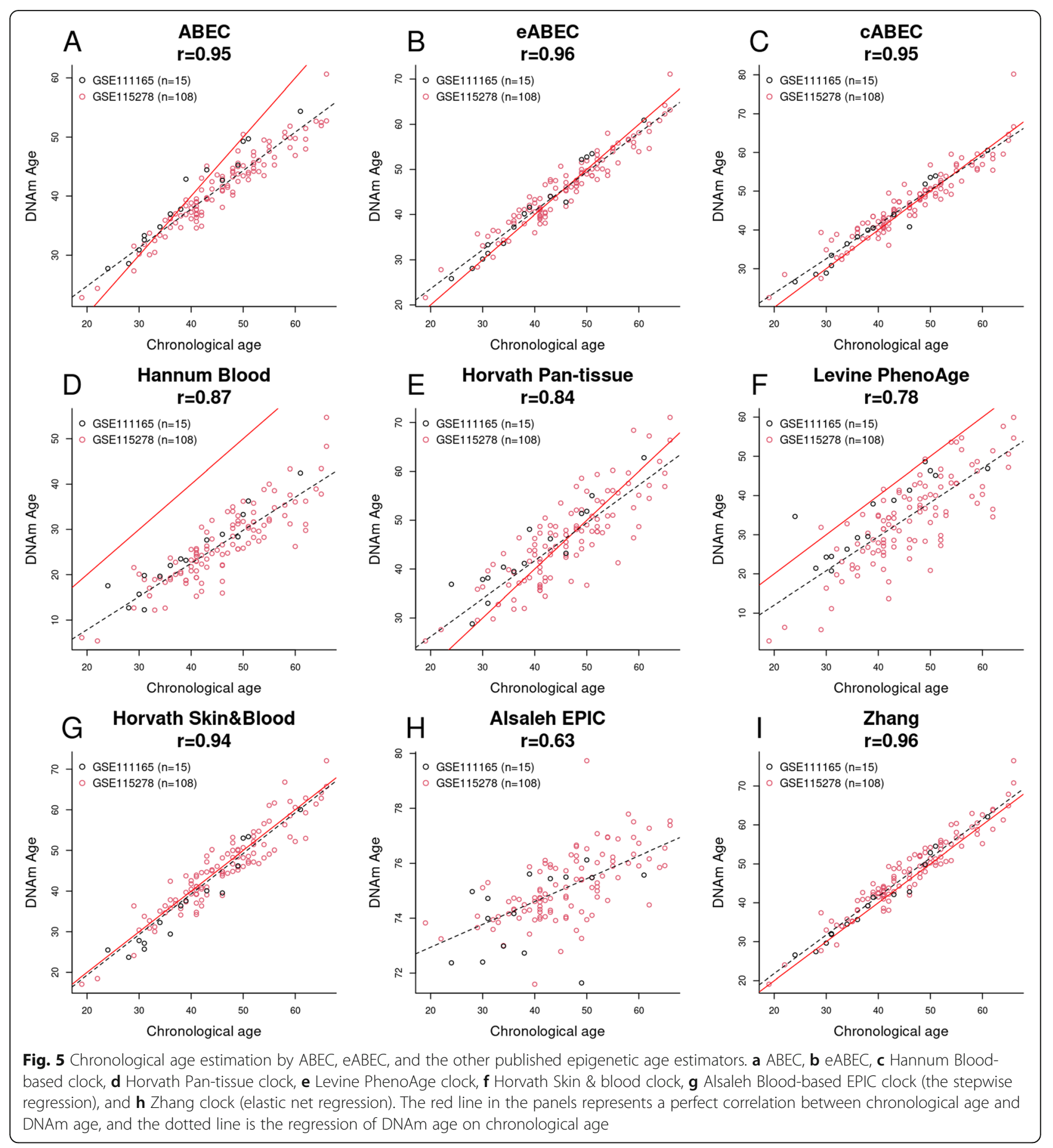

DNAm age on chronological age; i.e., the vertical distance between each dot and the dotted line in Figs. 2 and 3).

Our clocks, particularly eABEC, showed a systematic underestimation in older subjects, as was the case with the Horvath Pan-tissue clock and Hannum Blood-based clock in GSE132203 [37]. The systematic underestimation may be corrected by 1) adding more DNAm data of older subjects to the training set or 2) calibrating epigenetic clocks using a non-linear transformation (e.g., piecewise cubic regression (with a knot at 70) or smoothing spline of chronological age on DNAm age). However, we could not add more EPIC-derived DNAm data from older subjects (preferably subjects of European ancestry aged 70 to 80 years) to the training set for eABEC. We note that the underestimation in older subjects can cause EAA 


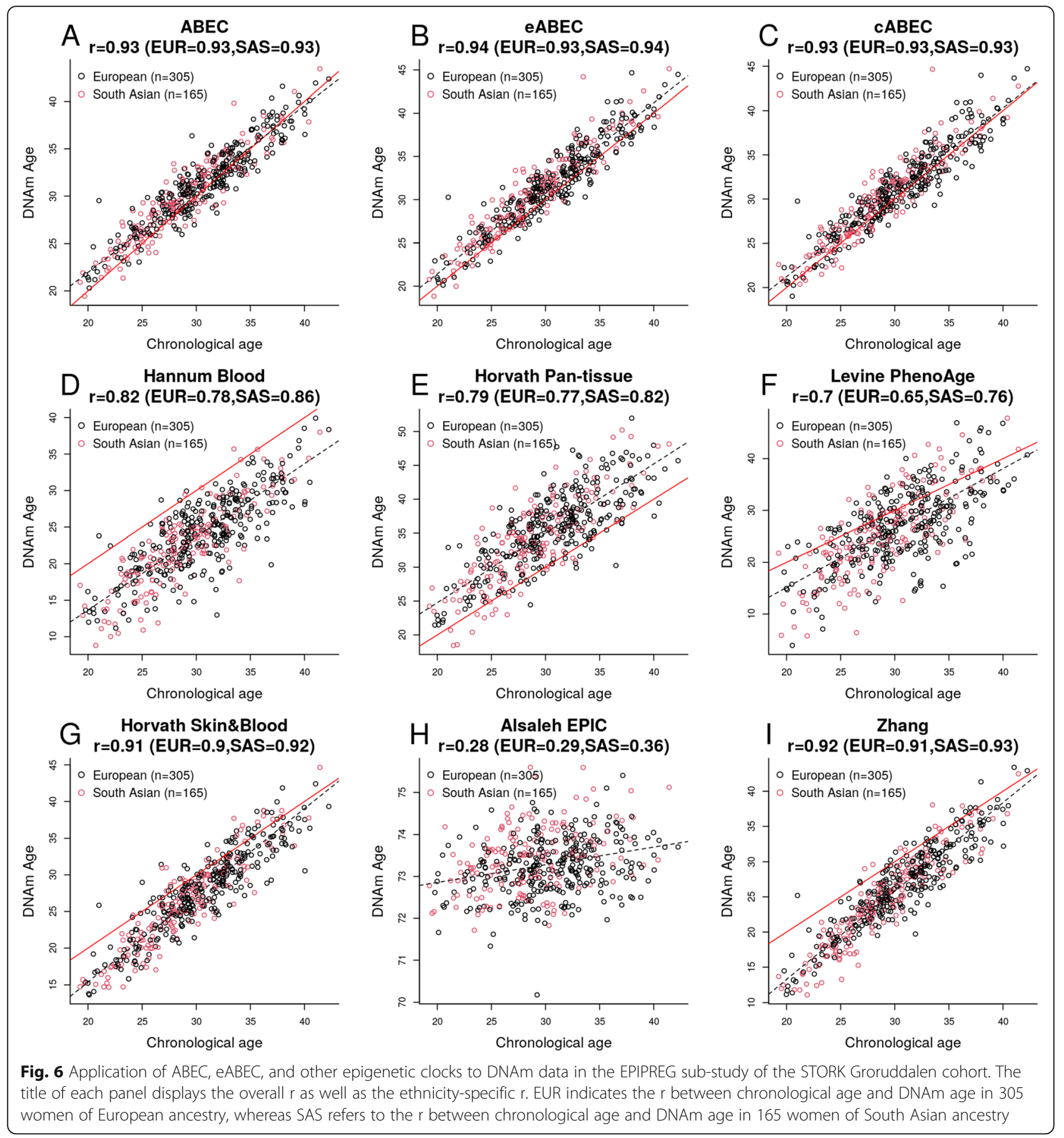

to be dependent on chronological age. Therefore, for other researchers who are interested in the association between EAA and a given phenotype, we recommend redefining EAA (e.g., regressing DNAm age on chronological age using a piecewise cubic regression or a smoothing spline rather than an ordinary linear regression) so that EAA is independent of chronological age.
Our eABEC may result in subtle differences in EAA across different ethnic groups, e.g., Supplementary File 2, S-Figure 2A. A hypothesis explaining this bias is that the CpGs included in eABEC may be located near SNPs with a low minor allele frequency [38]. The SNPs may influence the DNAm level at the CpGs if the minor allele frequencies at the SNPs differ across ethnicities. To address this point, we added 
SNP annotations generated by Zhou et al. [38] and McCartney et al. [39] to Supplementary File 1.

\section{Conclusion}

Three blood-based epigenetic clocks were developed to estimate adults' chronological age using EPIC-derived DNAm data. The precision of these clocks was high $(r>0.94)$ when validated in independent cohorts. The high level of precision was not explained by the broader genomic coverage of EPIC ( $>850,000 \mathrm{CpG}$ sites) but rather by the large training set $(n=2227)$ with a wide age-span (19 to 88 years).

\section{Methods}

\section{Study population}

$\mathrm{MoBa}$ is a nationwide pregnancy cohort study in which approximately 95,000 mothers, 75,000 fathers, and 114, 000 children were recruited from 1998 to 2008 across Norway [23]. The participants completed a series of questionnaires that are also linked to information from the Medical Birth Registry of Norway [23]. Peripheral wholeblood samples were collected from the mothers at the 17th week of gestation and at birth and from the fathers at the 17th week of gestation. Cord-blood samples were collected from newborns at birth [40, 41]. The precise chronological age in days at blood draw was calculated for the fathers and mothers. Further details on MoBa have been described in previous publications [23, 40-42]. We used data from a sub-study of MoBa (MoBa-START) with blood-based DNAm data on 2016 adults (mothers and fathers who were randomly selected among complete mother-father-newborn trios in MoBa).

GSE116339 is an epigenome-wide association study (EWAS) of polybrominated biphenyl in peripheral blood [25]. GSE111165 explored the difference in genomewide DNAm between brain and peripheral tissues (buccal, saliva, and blood) from epilepsy patients [26]. GSE115278 is an EWAS of insulin resistance, obesity, and metabolic complications [27, 43-45]. GSE132203 examined the association between DNAm and psychiatric or stress-related symptoms [28].

EPIPREG is nested within the STORK Groruddalen Cohort study (a population-based cohort, $n=823$, [24]). EPIPREG quantified DNAm in white blood cells, collected at the 28th week of gestation, from 480 women (312 of European ancestry and 168 of South Asian ancestry), using EPIC. In this study, we focused on 470 women (305 of European ancestry and 165 of South Asian ancestry) after excluding eight samples with low quality and two samples with an absolute EAA larger than 15 years. Further details of EPIPREG are described in Supplementary File 3 (S-Figure 7).

The age distributions of all the individuals included in the training and test sets can be found in Supplementary File 2 (S-Figure 5 and 6).

\section{Pre-processing of DNA methylation}

For MoBa-START, 500 nanograms of DNA stored in the MoBa Biobank (see Paltiel et al. [41] for further details of the storage of the biological samples) were shipped to LIFE \& BRAIN GmbH (Bonn, Germany). The samples were bisulfite converted and processed using the EZ-96DNA methylation-Lightning ${ }^{\text {mm }}$ MagPrep kit (Zymo Research, Irvine, USA) according to the manufacturer's instructions. The raw iDAT files were imported and processed using the RnBeads $\mathrm{R}$ package [46]. 44,210 probes with cross-hybridization [39], high detection $p$-value $(>0.01)$, and 16,117 probes near single-nucleotide polymorphisms (filtering.snp = "3") were excluded. The data were run in four batches and the exclusion criteria for removing probes were applied to each batch separately. Probes that were excluded from one batch were removed from all batches. The DNAm signals at the remaining probes were control-normalized and corrected for background noise using the wm.nasen and methylumi.noob options. Additionally, among a total of 2034 non-replicated samples, we excluded 18 samples that displayed low signal intensities and deviated (outliers) from the clusters formed by principal component analysis. The two probe chemistries (Type I and Type II probes) were normalized using Beta-mixture quantile normalization (BMIQ, [30]) using the wateRmelon $\mathrm{R}$ package [47]. In summary, the number of remaining probes was 790,213 (770,586 from autosomes and 19, 627 from sex-chromosomes).

For the DNAm data from GEO, we downloaded the iDAT files and used normal-exponential out-of-band (Noob, [29]) normalization in the minfi $\mathrm{R}$ package [48]. For the DNAm data from EPIPREG, we performed functional normalization (FunNorm, [31]) using the meffil $\mathrm{R}$ package [49]. Further details of the DNA extraction and quality control process of EPIPREG can be found in Supplementary File 3.

\section{Elastic net regression}

Penalized regressions (glmnet $\mathrm{R}$ package [50]) were used to develop the three ABECs. Chronological age in days was regressed on 770,586 autosomal CpGs that remained after quality control. The mixing parameter (alpha) was set to 0.5 and the shrinkage parameter (lambda) leading to the minimum mean square error was selected after 10fold cross-validation in the training set. Supplementary File 3 (S-Figure 8) includes cross-validation curves for lambda and alpha values. ABEC, eABEC and cABEC selected $1695 \mathrm{CpG}$ sites (lambda $=0.02884886), 1791 \mathrm{CpG}$ sites (lambda $=0.05281471$ ), and 1892 CpG sites (lambda $=0.0438477)$, respectively. Supplementary File 1 lists these $\mathrm{CpG}$ sites, their corresponding coefficients for $\mathrm{ABEC}$, eABEC, and $\mathrm{CABEC}$, and SNP annotations generated by Zhou et al. [38] and McCartney et al. [39]. 


\section{Comparison between EPIC-CpG clock and common CpG clock}

The implementation resembles bootstrapping conceptually. For each of the reduced sample sizes $(n=100,125,156$, 194, 243, 303, 378, 472, 589, 735, 918, 1145, 1430 and 1784; the determination of these values is detailed in Supplementary File 3), we first constructed five training sets by randomly selecting subjects from the full training set of eABEC $(n=2227)$. We made the sequence of the reduced sample sizes denser around 100 and sparser around 2227 because epigenetic clocks gradually improved their precision and accuracy when the training set was larger than 1145. On each training set, we trained two types of epigenetic clocks: one using all the CpGs on EPIC and the other using the CpGs common to EPIC and $450 \mathrm{~K}$. Next, we validated these clocks in the test set of eABEC $(n=485)$ and calculated $\mathrm{r}$ and MAD accordingly. The mgcv $\mathrm{R}$ package [51] was used to fit the smoothing splines in Fig. 4. Particularly, in Fig. 4a, we fit the smoothing splines of the Fisher's Z-transformed r values $\left(F(r)=0.5 * \log \left(\frac{1+r}{1-r}\right)\right)$ on the sample size, derived the confidence intervals and inversetransformed them.

\section{Availability of epigenetic clocks}

The estimated intercepts and coefficients for ABEC, eABEC, and cABEC can be found in Supplementary File 1.

The ABECs can be readily applied to any DNAm data using the following procedure: 1) generate a matrix of beta values ( $n$ individuals by $p$ CpG sites) using a background correction method, e.g., Noob (preferably) without any batch adjustment (Supplementary File 3), 2) select the $\mathrm{CpG}$ sites for the ABECs (Supplementary File 1) out of the matrix of beta values, 3) calculate the linear combination of the beta values at the selected CpG sites, and 4) add the estimated intercept (Supplementary File 1) to the linear combination.

\section{Supplementary Information}

The online version contains supplementary material available at https:/doi. org/10.1186/s12864-020-07168-8.

Additional file 1. This file includes $C p G$ sites for $A B E C$, eABEC, and $C A B E C$, their corresponding coefficients, overlap with the other published clocks, genomic locations, neighboring genes, presence in the Illumina HumanMethylation450K and $27 \mathrm{~K}$ array, and the SNP annotations generated by Zhou et al. [38] (with the suffix of "Zhou") and McCartney et al. [39] (with the suffix of "McCartney").

Additional file 2. This file includes 1) a figure displaying the age prediction of $C A B E C, 2$ ) a table containing the bootstrapped $95 \%$ confidence intervals for the $r$ values in Figs. 4, 5 and 6) figures displaying the age prediction of the ABECs and the other published clocks in EPIP REG and GSE132203, 4) a figure illustrating the regression-to-the-mean effect and 5) histograms displaying the age distribution of individuals in each cohort.

Additional file 3. This file includes 1) further details (sample selection, DNA extraction, and quality control) of EPIPREG, 2) cross-validation curves of mean squared error over lambda and alpha values for eABEC, 3) determination of the reduced sample sizes for Fig. 4, and 4) further information regarding batch adjustment in developing the ABECs.

\section{Abbreviations}

DNAm: DNA methylation; ABEC: Adult Blood EPIC Clock; eABEC: extended Adult Blood EPIC Clock; CABEC: common Adult Blood EPIC Clock; GEO: Gene Expression Omnibus; EPIC: Illumina MethylationEPIC BeadChip; 450 K: Illumina HumanMethylation $450 \mathrm{~K}$ BeadChip; r: The Pearson correlation coefficient; MAD: Median absolute deviation; BMIQ: Beta-mixture quantile dilation; MoBa: Norwegian Mother, Father and Child Cohort Study; START: Study of Assisted Reproductive Technology; EPIPREG: Epigenetics in Pregnancy; EAA: Epigenetic age acceleration

\section{Acknowledgements}

MoBa is supported by the Norwegian Ministry of Health and Care Services and the Ministry of Education and Research. We are grateful to all the families in Norway who take part in this on-going cohort study. We also appreciate all the GEO submitters for making their DNAm data publically available.

\section{Authors' contributions}

$Y L$ developed the three ABECs and wrote the manuscript. HEN, CP, and RL performed quality control on the DNAm data from MoBa-START. CS initiated EPIPREG and leads the EPIPREG group. GHM performed the quality control and normalization of the EPIPREG samples. SL- $\varnothing$ validated all the epigenetic clocks in the DNAm data from EPIPREG. LS participated in data acquisition in the STORK Groruddalen study. RBP and LCG performed chip analysis for EPIP REG. HKG provided statistical interpretations. SEH and PM initiated START and $\mathrm{MoBa}$, respectively. All the authors contributed to data interpretation, provided overall scientific input, and revised the manuscript. The author(s) read and approved the final manuscript.

\section{Funding}

This work is supported in part by a grant from the Research Council of Norway (RCN) to AJ, JRH, PM, and HKG (grant 262043). The work of KLH, HEN, CP, RL, MCM, HKG, JRH, PM, SEH, AJ, and JB was supported in part by RCN through its Centres of Excellence funding scheme, project number 262700 (awarded to SEH and PM). GHM is supported by RCN (Postdoctoral mobility research grant 287198). The funding body played no role in the design of the study, analysis or interpretation of data, nor in writing the manuscript.

\section{Availability of data and materials}

The MoBa data can be accessed by applying directly to the Norwegian Institute of Public Health, http://www.fhi.no/en/. The EPIPREG data can be accessed by contacting Dr. Christine Sommer, Oslo University Hospital, https://www.oslodiabetes.no/christine-sommer. The publicly available DNAm data in this study (accession numbers: GSE116339, GSE111165, GSE115278, and GSE132203) are accessible on the GEO repository, https://www.ncbi.nlm. nih.gov/geo/. Public access to the GEO repository is open, and thus administrative permission to access and use the data is not needed.

\section{Ethics approval and consent to participate}

This study was approved by the Regional Committees for Medical and Health Research Ethics (REK) South-East (2017/1362) in Norway. Data collec tion by MoBa was carried out in accordance with the Norwegian Data Protection Agency after securing approval from REK. The participation in the STORK Groruddalen study was based on informed written consent, and the study and its sub-study, EPIPREG, were approved by the REK South-East (2015/1035).

\section{Consent for publication}

Written consents were obtained from the MoBa and STORK participants.

\section{Competing interests}

The authors declare no conflicts of interest.

\section{Author details}

${ }^{1}$ Department of Genetics and Bioinformatics, Norwegian Institute of Public Health, Oslo, Norway. ${ }^{2}$ Institute of Health and Society, Faculty of Medicine, 
University of Oslo, Oslo, Norway. ${ }^{3}$ Centre for Fertility and Health, Norwegian Institute of Public Health, Oslo, Norway. ${ }^{4}$ Department of Global Public Health and Primary Care, University of Bergen, N-5020 Bergen, Norway. ${ }^{5}$ Deepinsight, Karl Johans gate 8, Oslo, Norway. ${ }^{6}$ Oslo Centre for Biostatistics and Epidemiology, Section for Research Support, Oslo University Hospital, Oslo, Norway. ${ }^{7}$ Department of Medical Genetics, Oslo University Hospital, Oslo, Norway. ${ }^{8}$ PharmaTox Strategic Research Initiative, School of Pharmacy, Faculty of Mathematics and Natural Sciences, University of Oslo, Oslo, Norway. ${ }^{9}$ Department of Internal Medicine, Akershus University Hospital, Kongsvinger, Norway. ${ }^{10}$ Department of transplantation medicine, Institute of Clinical medicine, University of Oslo, Oslo, Norway. ${ }^{11}$ Institute of Clinical Medicine, Faculty of Medicine, University of Oslo, Oslo, Norway. ${ }^{12}$ The University of Queensland Diamantina Institute, University of Queensland, Woolloongabba, QLD 4102, Australia. ${ }^{13}$ K.G. Jebsen Center for Genetic Epidemiology, Department of Public Health and Nursing, Norwegian University of Science and Technology, Trondheim, Norway. ${ }^{14}$ Population Health Science, Bristol Medical School, University of Bristol, Bristol, UK ${ }^{15}$ Department of Clinical Sciences, Clinical Research Centre, Lund University, Malmö, Sweden. ${ }^{16}$ Finnish Institute of Molecular Medicine, Helsinki University, Helsinki, Finland. ${ }^{17}$ Department of Pediatric and Adolescents Medicine, Akershus University Hospital, Lørenskog, Norway. ${ }^{18}$ Institute of Clinical Medicine, University of Oslo, Campus AHUS, Lørenskog, Norway.

${ }^{19}$ Department of Endocrinology, Morbid Obesity and Preventive Medicine, Oslo University Hospital, Oslo, Norway. ${ }^{20}$ MRC Integrative Epidemiology Unit at the University of Bristol, Bristol, UK. ${ }^{21}$ Division for Infection Control and Environmental Health, Department of Infectious Disease Epidemiology and Modelling, Norwegian Institute of Public Health, Oslo, Norway.

Received: 12 March 2020 Accepted: 20 October 2020

Published online: 27 October 2020

\section{References}

1. Baker GT 3rd, Sprott RL. Biomarkers of aging. Exp Gerontol. 1988;23(4-5): 223-39.

2. Warner HR. Current status of efforts to measure and modulate the biological rate of aging. J Gerontol A Biol Sci Med Sci. 2004;59(7):692-6.

3. Horvath S, Oshima J, Martin GM, Lu AT, Quach A, Cohen H, Felton S, Matsuyama M, Lowe D, Kabacik S, et al. Epigenetic clock for skin and blood cells applied to Hutchinson Gilford Progeria syndrome and ex vivo studies. Aging (Albany NY). 2018;10(7):1758-75.

4. Jylhava J, Pedersen NL, Hagg S. Biological age predictors. EBioMedicine. 2017;21:29-36

5. Melzer D, Pilling LC, Ferrucci L. The genetics of human ageing. Nat Rev Genet. 2020;21(2):88-101

6. Bell CG, Lowe R, Adams PD, Baccarelli AA, Beck S, Bell JT, Christensen BC, Gladyshev VN, Heijmans BT, Horvath S, et al. DNA methylation aging clocks: challenges and recommendations. Genome Biol. 2019;20(1):249.

7. Lopez-Otin C, Blasco MA, Partridge L, Serrano M, Kroemer G. The hallmarks of aging. Cell. 2013;153(6):1194-217.

8. Aubert G, Lansdorp PM. Telomeres and aging. Physiol Rev. 2008:88(2):557-79.

9. Johnson LC, Parker K, Aguirre BF, Nemkov TG, D'Alessandro A, Johnson SA, Seals DR, Martens CR. The plasma metabolome as a predictor of biological aging in humans. Geroscience. 2019;41(6):895-906.

10. Bocklandt S, Lin W, Sehl ME, Sanchez FJ, Sinsheimer JS, Horvath S, Vilain E. Epigenetic predictor of age. PLoS One. 2011;6(6):e14821.

11. Jagger A, Shimojima Y, Goronzy JJ, Weyand CM. Regulatory T cells and the immune aging process: a mini-review. Gerontology. 2014;60(2):130-7.

12. Lehallier B, Gate D, Schaum N, Nanasi T, Lee SE, Yousef H, Moran Losada P, Berdnik D, Keller A, Verghese J, et al. Undulating changes in human plasma proteome profiles across the lifespan. Nat Med. 2019;25(12):1843-50.

13. Odamaki T, Kato K, Sugahara H, Hashikura N, Takahashi S, Xiao JZ, Abe F, Osawa R. Age-related changes in gut microbiota composition from newborn to centenarian: a cross-sectional study. BMC Microbiol. 2016;16:90.

14. Dugue PA, Bassett JK, Joo JE, Jung CH, Ming Wong E, Moreno-Betancur M, Schmidt D, Makalic E, Li S, Severi G, et al. DNA methylation-based biological aging and cancer risk and survival: pooled analysis of seven prospective studies. Int J Cancer. 2018;142(8):1611-9.

15. Levine ME, Hosgood HD, Chen B, Absher D, Assimes T, Horvath S. DNA methylation age of blood predicts future onset of lung cancer in the women's health initiative. Aging (Albany NY). 2015;7(9):690-700.
16. Levine ME, Lu AT, Quach A, Chen BH, Assimes TL, Bandinelli S, Hou L, Baccarelli AA, Stewart JD, Li Y, et al. An epigenetic biomarker of aging for lifespan and healthspan. Aging (Albany NY). 2018;10(4):573-91.

17. Lu AT, Quach A, Wilson JG, Reiner AP, Aviv A, Raj K, Hou L, Baccarelli AA, Li $Y$, Stewart JD, et al. DNA methylation GrimAge strongly predicts lifespan and healthspan. Aging (Albany NY). 2019;11(2):303-27.

18. Marioni RE, Shah S, McRae AF, Chen BH, Colicino E, Harris SE, Gibson J, Henders AK, Redmond P, Cox SR, et al. DNA methylation age of blood predicts all-cause mortality in later life. Genome Biol. 2015;16:25.

19. Hannum G, Guinney J, Zhao L, Zhang L, Hughes G, Sadda S, Klotzle B, Bibikova M, Fan JB, Gao Y, et al. Genome-wide methylation profiles reveal quantitative views of human aging rates. Mol Cell. 2013;49(2):359-67.

20. Horvath S. DNA methylation age of human tissues and cell types. Genome Biol. 2013;14(10):R115.

21. Pidsley R, Zotenko E, Peters TJ, Lawrence MG, Risbridger GP, Molloy P, Van Djik S, Muhlhausler B, Stirzaker C, Clark SJ. Critical evaluation of the Illumina MethylationEPIC BeadChip microarray for whole-genome DNA methylation profiling. Genome Biol. 2016;17(1):208.

22. Alsaleh $\mathrm{H}$, Haddrill PR. Identifying blood-specific age-related DNA methylation markers on the Illumina MethylationEPIC(R) BeadChip. Forensic Sci Int. 2019;303:109944.

23. Magnus P, Birke C, Vejrup K, Haugan A, Alsaker E, Daltveit AK, Handal M, Haugen M, Hoiseth G, Knudsen GP, et al. Cohort profile update: the Norwegian mother and child cohort Study (MoBa). Int J Epidemiol. 2016;45(2):382-8.

24. Jenum AK, Sletner L, Voldner N, Vangen S, Morkrid K, Andersen LF, Nakstad B, Skrivarhaug T, Rognerud-Jensen OH, Roald B, et al. The STORK Groruddalen research programme: a population-based cohort study of gestational diabetes, physical activity, and obesity in pregnancy in a multiethnic population. Rationale, methods, study population, and participation rates. Scand J Public Health. 2010;38(5 Suppl):60-70.

25. Curtis SW, Cobb DO, Kilaru V, Terrell ML, Kennedy EM, Marder ME, Barr DB, Marsit CJ, Marcus M, Conneely KN, et al. Exposure to polybrominated biphenyl (PBB) associates with genome-wide DNA methylation differences in peripheral blood. Epigenetics. 2019;14(1):52-66.

26. Braun PR, Han S, Hing B, Nagahama Y, Gaul LN, Heinzman JT, Grossbach AJ, Close L, Dlouhy BJ, Howard MA 3rd, et al. Genome-wide DNA methylation comparison between live human brain and peripheral tissues within individuals. Transl Psychiatry. 2019;9(1):47.

27. Arpon A, Milagro Fl, Santos JL, Garcia-Granero M, Riezu-Boj J, Martinez JA. Interaction among sex, aging, and epigenetic processes concerning visceral fat, insulin resistance, and Dyslipidaemia. Front Endocrinol (Lausanne). 2019;10:496.

28. Kilaru V. GSE132203, DNA Methylation (EPIC) from the Grady Trauma Project; 2019.

29. Triche TJ Jr, Weisenberger DJ, Van Den Berg D, Laird PW, Siegmund KD. Low-level processing of Illumina Infinium DNA methylation BeadArrays. Nucleic Acids Res. 2013;41(7):e90.

30. Teschendorff AE, Marabita F, Lechner M, Bartlett T, Tegner J, Gomez-Cabrero D, Beck S. A beta-mixture quantile normalization method for correcting probe design bias in Illumina Infinium 450 k DNA methylation data. Bioinformatics. 2013;29(2):189-96.

31. Fortin JP, Labbe A, Lemire M, Zanke BW, Hudson TJ, Fertig EJ, Greenwood CM, Hansen KD. Functional normalization of 450k methylation array data improves replication in large cancer studies. Genome Biol. 2014;15(12):503.

32. Zou H, Hastie T. Regularization and variable selection via the elastic net. J R Stat Soc B. 2005;67(2):301-20.

33. Barrett T, Wilhite SE, Ledoux P, Evangelista C, Kim IF, Tomashevsky M, Marshall KA, Phillippy KH, Sherman PM, Holko M, et al. NCBI GEO: archive for functional genomics data sets--update. Nucleic Acids Res. 2013;41(Database issue):D991-5.

34. Zhang Q, Vallerga CL, Walker RM, Lin T, Henders AK, Montgomery GW, He J, Fan D, Fowdar J, Kennedy M, et al. Improved precision of epigenetic clock estimates across tissues and its implication for biological ageing. Genome Med. 2019;11(1):54.

35. Thurman RE, Rynes E, Humbert R, Vierstra J, Maurano MT, Haugen E, Sheffield NC, Stergachis AB, Wang H, Vernot B, et al. The accessible chromatin landscape of the human genome. Nature. 2012;489(7414):75-82.

36. Andersson R, Gebhard C, Miguel-Escalada I, Hoof I, Bornholdt J, Boyd M, Chen $Y$, Zhao X, Schmidl C, Suzuki T, et al. An atlas of active enhancers across human cell types and tissues. Nature. 2014;507(7493):455-61.

37. El Khoury LY, Gorrie-Stone T, Smart M, Hughes A, Bao Y, Andrayas A, Burrage J, Hannon E, Kumari M, Mill J, et al. Systematic underestimation of the epigenetic clock and age acceleration in older subjects. Genome Biol. 2019;20(1):283. 
38. Zhou W, Laird PW, Shen H. Comprehensive characterization, annotation and innovative use of Infinium DNA methylation BeadChip probes. Nucleic Acids Res. 2017:45(4):e22.

39. McCartney DL, Walker RM, Morris SW, McIntosh AM, Porteous DJ, Evans KL. Identification of polymorphic and off-target probe binding sites on the Illumina Infinium MethylationEPIC BeadChip. Genom Data. 2016;9:22-4.

40. Ronningen KS, Paltiel L, Meltzer HM, Nordhagen R, Lie KK, Hovengen R, Haugen M, Nystad W, Magnus P, Hoppin JA. The biobank of the Norwegian mother and child cohort Study: a resource for the next 100 years. Eur J Epidemiol. 2006;21(8):619-25.

41. Paltiel L, Anita H, Skjerden T, Harbak K, Bækken S, Kristin SN, Knudsen GP, Magnus P. The biobank of the Norwegian Mother and Child Cohort Studypresent status. Norsk epidemiologi. 2014;24:1-2.

42. Magnus P, Irgens LM, Haug K, Nystad W, Skjaerven R, Stoltenberg C, MoBa Study G. Cohort profile: the Norwegian mother and child cohort Study (MoBa). Int J Epidemiol. 2006;35(5):1146-50.

43. Salas-Perez F, Ramos-Lopez O, Mansego ML, Milagro Fl, Santos JL, Riezu-Boj $\mathrm{Jl}$, Martinez JA. DNA methylation in genes of longevity-regulating pathways: association with obesity and metabolic complications. Aging (Albany NY). 2019;11(6):1874-99.

44. Arpon A, Milagro Fl, Ramos-Lopez O, Mansego ML, Santos JL, Riezu-Boj Jl, Martinez JA. Epigenome-wide association study in peripheral white blood cells involving insulin resistance. Sci Rep. 2019;9(1):2445.

45. Arpon A, Milagro FI, Ramos-Lopez O, Mansego ML, Riezu-Boj Jl, Martinez JA, Project M. Methylome-Wide Association Study in Peripheral White Blood Cells Focusing on Central Obesity and Inflammation. Genes (Basel). 2019; 10(6):444.

46. Muller F, Scherer M, Assenov Y, Lutsik P, Walter J, Lengauer T, Bock C. RnBeads 2.0: comprehensive analysis of DNA methylation data. Genome Biol. 2019;20(1):55.

47. Pidsley R, YW CC, Volta M, Lunnon K, Mill J, Schalkwyk LC. A data-driven approach to preprocessing Illumina $450 \mathrm{~K}$ methylation array data. BMC Genomics. 2013:14:293.

48. Aryee MJ, Jaffe AE, Corrada-Bravo H, Ladd-Acosta C, Feinberg AP, Hansen KD, Irizarry RA. Minfi: a flexible and comprehensive bioconductor package for the analysis of Infinium DNA methylation microarrays. Bioinformatics. 2014:30(10):1363-9.

49. Min JL, Hemani G, Davey Smith G, Relton C, Suderman M. Meffil: efficient normalization and analysis of very large DNA methylation datasets. Bioinformatics. 2018;34(23):3983-9.

50. Friedman J, Hastie T, Tibshirani R. Regularization paths for generalized linear models via coordinate descent. J Stat Softw. 2010;33(1):1-22.

51. Wood SN. Fast stable restricted maximum likelihood and marginal likelihood estimation of semiparametric generalized linear models. J R Stat Soc B. 2011;73(1):3-36

\section{Publisher's Note}

Springer Nature remains neutral with regard to jurisdictional claims in published maps and institutional affiliations.

Ready to submit your research? Choose BMC and benefit from:

- fast, convenient online submission

- thorough peer review by experienced researchers in your field

- rapid publication on acceptance

- support for research data, including large and complex data types

- gold Open Access which fosters wider collaboration and increased citations

- maximum visibility for your research: over $100 \mathrm{M}$ website views per year

At $\mathrm{BMC}$, research is always in progress.

Learn more biomedcentral.com/submissions 